\title{
Nuevos métodos de intervención en el aula y en el espacio virtual: glosario y juego de rol
}

\author{
Rocío Chamizo SÁNCHEZ \\ Universidad de Málaga \\ rcs@uma.es \\ María Jesús Fernández ToRres \\ Universidad de Málaga \\ mariajesusfernandez@uma.es
}

\section{Resumen}

En este artículo, presentamos los resultados de una experiencia docente desarrollada en el marco del Proyecto de Innovación Educativa titulado "Aplicación de las TIC y el Campus Virtual para el aprendizaje cooperativo", llevado a cabo en materias de Comunicación, tanto de grado/licenciatura como de postgrado, entre los años 2010 y 2012. Dicha experiencia ha pretendido identificar los beneficios de combinar la docencia presencial con recursos propios de las TIC, a partir del desarrollo de actividades didácticas centradas en el aprendizaje cooperativo.

Palabras clave: aprendizaje cooperativo; aprendizaje colaborativo; glosario colaborativo; juego de rol; entornos virtuales

New Methods for working both in classroom and on the virtual space: glossary and role play

\begin{abstract}
This article shows the outcomes of an teaching experience implemented in the frame of the educative innovation project call: "TIC Management on the virtual campus for the cooperative learning". It was carried out on different subjects of Communication Science, both on degree and post degree from 2010 to 2012. This project had intended to find out the benefits of the combination between face to face learning and resources from T.I.C. in connection with the developments of didactic activities based on cooperative learning.
\end{abstract}

Key words: Cooperative learning; collaborative learning; collaborative glossary; rol play; virtual environment

Referencia normalizada:

Chamizo Sánchez, R. y Fernández Torres, M. J. (2013) Nuevos métodos de intervención en el aula y en el espacio virtual: glosario y juego de rol. Historia y Comunicación Social. Vol. 18. No Especial Diciembre. Págs. 753-764.

Sumario: 1. Introducción. 2. Estado de la cuestión. 3. Descripción de la experiencia: metodología y resultados. 4. Conclusiones. 5. Referencias Bibliográficas. 


\section{Introducción}

Las instituciones universitarias están asistiendo a un proceso de convergencia que plantea un nuevo modelo educativo que prioriza la capacidad de "aprender a aprender" en espacios participativos en los que se fomente el protagonismo del estudiante en su aprendizaje y el trabajo en equipo.

En este nuevo contexto, las Tecnologías de la Información y la Comunicación (TIC) se convierten en una pieza fundamental del cambio y para el cambio. El desarrollo de entornos virtuales como nuevos espacios para el aprendizaje colabora de forma decisiva en la adopción del nuevo modelo educativo, proporcionando, entre otros aspectos (Pérez Casales et al., 2008): una mayor riqueza del proceso de formación, con el empleo de nuevas herramientas (foros, wikis...) que fomentan el trabajo en equipo y la consecución de retos comunes; una mayor motivación por el aprendizaje, proporcionando al estudiante la flexibilidad para poder decidir cuándo, dónde y hasta cómo formarse; una mayor comunicación entre los agentes educativos y el seguimiento del proceso de enseñanza-aprendizaje, tanto por parte del docente como por parte del alumno.

La experiencia docente que presentamos en esta ponencia forma parte de un Proyecto de Innovación Educativa titulado "Aplicación de las TIC y el Campus Virtual para el aprendizaje cooperativo", llevado a cabo entre los años 2010 y 2012, en asignaturas de Comunicación, tanto de grado/licenciatura como de postgrado, en su mayoría adaptadas al EEES. El proyecto ha combinado la docencia presencial con recursos propios de las TIC y ha perseguido desarrollar actividades didácticas centradas en el aprendizaje cooperativo.

Moodle, uno de los entornos virtuales más usados en la actualidad, ha sido la plataforma interactiva de enseñanza-aprendizaje utilizada en el proyecto. Este entorno interactivo se ha utilizado como complemento a la clase presencial, proporcionando material de apoyo, desarrollando actividades cooperativas que fomenten el pensamiento crítico, el trabajo en equipo y refuercen el contenido impartido en el aula, permitiendo una comunicación en línea entre los diferentes agentes del proceso, profesor y estudiantes.

De las distintas actividades puestas en práctica en el entorno virtual de las materias implicadas, vamos a centrarnos en la explicación de dos de ellas: el glosario y el juego de rol. Dos actividades que nos han permitido, por una parte, la asimilación de conceptos y la creación de contenidos y, por otra, la comprensión y adopción de las rutinas profesionales. 


\section{Estado de la cuestión}

Si revisamos la literatura de pedagogía son diferentes los tipos de aprendizaje que pueden encontrarse, si bien, en este trabajo se abordarán las posiciones expuestas en cuanto a los términos de aprendizaje colaborativo y aprendizaje cooperativo, donde diversos autores los ven como semejantes y, otros, como contrapuestos. Tanto uno como otro implican trabajo en equipo. El criterio más generalizado que se utiliza para diferenciarlos, está relacionado con el grado de responsabilidad del estudiante y del profesor en el aprendizaje. Así, Panitz (1998) plantea: "Cada paradigma representa un extremo de un espectro del proceso enseñanza-aprendizaje, que va de ser altamente estructurado por el profesor (cooperativo), hasta dejar la responsabilidad del aprendizaje principalmente en el estudiante (colaborativo)".

Otro criterio es que en el aprendizaje colaborativo todos y cada uno de los participantes del grupo intervienen en todas y cada una de las partes del proyecto a resolver; mientras que en el modelo cooperativo, cada uno de los integrantes del grupo, tiene destinada una tarea específica dentro del proyecto o problema, realizando en este caso un trabajo más individual. Se puede decir que:

- En el Aprendizaje Cooperativo el profesor es quien propone un problema y determina el rol de cada estudiante para su solución, por lo que cada alumno se responsabiliza de una parte de la solución de la tarea; mientras que en el Aprendizaje Colaborativo, el profesor propone la actividad y se transforma en un guía para sus pupilos pero son los estudiantes los responsables del resultado. Tampoco el docente se encarga de definir los roles.

- En el Método Colaborativo se requiere de una preparación más avanzada de los estudiantes mientras que en el cooperativo la heterogeneidad del alumnado puede ser mayor.

- El fin último del Aprendizaje Cooperativo es la construcción de nuevas ideas con la contribución de pares lo que favorece muy especialmente a aquellos que cuentan con dificultades y enriquece a los más aventajados. En cambio, el Aprendizaje Colaborativo tiene como objetivo que cada estudiante desarrolle nuevas ideas y cree en conjunto con los demás, es decir, que cada estudiante aporte lo mejor de sí a un fin común y no necesariamente debe abarcar a aquellos alumnos con dificultades en el aprendizaje.

Es evidente que las transformaciones sufridas por la sociedad actual suponen que deba existir un cambio significativo en la concepción del aprendizaje y de las funciones a cubrir por las distintas instituciones educativas. Lo importante ya no debe ser la simple adquisición y repetición de información, sino el dominio de otras habilidades que lleven al alumno a pensar, a seleccionar sus rutas de aprendizaje y aprender a aprender. Las TIC aumentan la oferta educativa para los estudiantes y ya es posible dotarlos de nuevos modelos de enseñanza. 
El profesor también debe cambiar sus rutinas de trabajo ya que va a jugar un papel muy importante en el aula en lo que a diseño de materiales, recursos y medios se refiere, adaptándolos a las características de sus estudiantes. No será, por tanto, un mero transmisor de información sino que tendrá que desempeñar otras funciones como consultor, creador de medios, facilitador de información, diseñador de situaciones de aprendizaje, moderador, tutor, así como, evaluador continuo.

Se puede decir que para aprender son necesarios tres actos imprescindibles: observar, estudiar y practicar; así como cuatro factores fundamentales: inteligencia, conocimientos previos, experiencia y motivación.

Ortega et al. (2008) inciden en la importancia de aprender en cooperación con otros para: resolver problemas; tomar iniciativas y madurar en las relaciones con otros; planificar y realizar actividades en grupo; adecuar los objetivos e intereses propios a los del resto del grupo; proponer normas y respetarlas; entender y respetar opiniones e intereses diferentes al propio; comportarse de acuerdo a los valores y normas que rigen las relaciones entre personas valorando su importancia. Según Johnson et al. (1999), el trabajo en conjunto para alcanzar objetivos comunes produce logros superiores y mayor productividad que el trabajo individual. También señala que el aprendizaje cooperativo favorece un mayor uso de estrategias superiores de razonamiento y pensamiento crítico que el aprendizaje competitivo e individualista.

Asimismo, tanto en uno como en otro, el trabajo del profesor con el grupo implica una transformación notable en la estructuración y concepción de la enseñanza. Para Ortega et al. (2008) la tarea del docente es imprescindible y cumple, a su entender, tres roles fundamentales: mediador, observador y facilitador de la autonomía en el aprendizaje.

\section{Descripción de la experiencia: metodología y resultados}

\subsection{El glosario como herramienta educativa}

La actividad se desarrolló en el marco del módulo "La imagen en el sector turístico" del master oficial en Gestión Estratégica e Innovación en Comunicación que se imparte en la Universidad de Málaga desde el año 2009. En esta ocasión, la actividad se llevó a cabo durante el curso académico 2011-2012, contando con un total de treinta y ocho estudiantes matriculados.

El glosario es una de las herramientas disponibles en la plataforma de e-learning Moodle que permite la creación de un diccionario o enciclopedia a partir de la aportación de conceptos. En nuestro caso, son los estudiantes quienes, con las debidas directrices del profesor, que actúa en calidad de facilitador, introducen las definiciones para crear un glosario en torno a la materia en cuestión. 
En este sentido, la finalidad de esta actividad es construir, entre todos, un material que sirva de apoyo al contenido del módulo y que recoja los principales conceptos e ideas trabajados durante el curso. Se trata de obtener un material de calidad producto de la participación de todos los usuarios. El diseño de esta actividad encierra, en primer lugar, un trabajo individual de recuperación de información, comprensión, asimilación y reflexión por parte del alumno, y, en segundo lugar, un trabajo colaborativo de construcción de conceptos y valoración de ideas. Se trata, en definitiva, de una actividad programada que responde a la teoría de la formación por etapas a la que se refiere Galperin (1995), citado por Pérez Casales et al. (2008), basada en el desarrollo de capacidades y valores a través de fases secuenciales con objetivos bien definidos.

\subsubsection{Descripción de la actividad}

Objetivos planteados. El uso de esta actividad en el módulo "Imagen en el sector turístico" ha pretendido: Fomentar la participación y el debate en el proceso de formación mediante el empleo de herramientas propias de los nuevos escenarios de aprendizaje; estimular a un estudiante familiarizado con el uso de las Nuevas Tecnologías en su proceso de formación; afianzar los conceptos surgidos a lo largo del módulo y extender el debate y la reflexión más allá del aula, de tal forma que permita a los alumnos profundizar en las ideas trabajadas en clase y asimilar el contenido y dotar al alumno de un material complementario, elaborado por ellos y revisado por el docente, que recoja los principales conceptos trabajados en el módulo y sirva de apoyo y consulta en su formación.

Recursos empleados y duración de la actividad. Hemos precisado de una plataforma interactiva de e-learning, en nuestro caso, del entorno Moodle. Para desarrollar la actividad, el alumno necesitará de conexión a Internet y de la lectura de artículos, informes y estudios que serán facilitados por el docente a través del campus virtual de la asignatura. La actividad se desarrollará a lo largo de cuatro semanas, coincidiendo con la duración del módulo.

Diseño de la actividad. La actividad fue presentada a los estudiantes del módulo con el nombre "Definiendo conceptos". La tarea consistía en la construcción de un diccionario o enciclopedia a partir de los conceptos introducidos por los estudiantes y revisados por el profesor. El ejercicio sólo exigía la elaboración de un único glosario que actuaba de glosario principal y que, tras la conveniente valoración positiva del profesor, se convertiría, al finalizar el tiempo para su desarrollo, en el glosario definitivo de la materia. El ejercicio se inicia con la aportación, por parte del docente, de material relacionado con la materia impartida (de texto y/o audiovisual) a través del campus virtual del módulo. Un contenido que constituye un complemento y una ampliación a los conocimientos transmitidos en el aula y que supone una continuidad del proceso de enseñanza-aprendizaje iniciado.

A partir del contenido disponible, el alumno comienza un proceso de lectura, comprensión, reflexión y asimilación de la información aportada, así como, de 
búsqueda activa de contenido relacionado. Tras esta etapa, intervendrá en el glosario incorporando definiciones, descripciones y/o clasificaciones en torno a los conceptos trabajados. En todo momento, tendrá la oportunidad de añadir comentarios a las entradas de otros compañeros con el objetivo de matizar, ampliar $u$ ofrecer un nuevo punto de vista que enriquezca el concepto inicial. Por otro lado, no se permite duplicar conceptos, tan sólo, realizar aportaciones a modo de comentarios a los ya existentes.

A lo largo de las cuatro semanas que dura la construcción del diccionario, las entradas serán aprobadas por defecto, es decir, las aportaciones de los alumnos quedan incorporadas directamente al glosario sin mediar una revisión previa del docente. El objetivo es que los propios estudiantes, a través de sus intervenciones, adviertan los posibles aciertos y/o errores. Las distintas entradas efectuadas no sólo se trabajarán a través del campus virtual, sino, también, si el profesor lo estima conveniente, en las horas presenciales de clase, con la finalidad de aclarar posibles confusiones.

Por otra parte, las entradas, en principio, no podrán ser editadas por el usuario, hasta que, realizadas las observaciones pertinentes y debatidas lo suficientemente por los participantes, el docente ofrezca la posibilidad a los autores de editar los conceptos para ser corregidos, matizados y/o ampliados, de tal forma que estas definiciones sean las que formen, parte del glosario final. Finalmente, el glosario podrá ser impreso para convertirse en material didáctico de apoyo.

Función del docente y el discente. A lo largo de la construcción del glosario, la función del profesor será la de facilitador y guía, iniciando la actividad con la aportación de material de apoyo y controlando el desarrollo de la misma a través de la revisión de las intervenciones del alumnado y aclarando posibles dudas. En cuanto al discente, éste deberá adoptar un rol activo tanto en la recuperación de información como en la elaboración del diccionario. La actividad a desarrollar exigirá introducir un mínimo de cinco definiciones por alumno.

Sistema de evaluación de la actividad. El trabajo se valorará considerando el número total de aportaciones realizadas al glosario por cada estudiante, tanto por sus definiciones propias como por los comentarios al resto de entradas, y el número de aportaciones que supere la revisión del profesor, para formar parte del glosario final, por la pertinencia de la misma con el contenido de la materia y su adecuada y completa formulación.

\subsubsection{Resultados}

Finalizada la actividad, se solicitó a los participantes cumplimentar un cuestionario anónimo para conocer su opinión respecto al uso de esta herramienta y la aportación a su aprendizaje. Los resultados han sido bastante satisfactorios. La totalidad de los usuarios de esta herramienta coinciden en la utilidad de la misma para su aprendizaje, encontrando más aspectos positivos que negativos en su empleo.

Respecto a los aspectos positivos destacados por el alumnado, valoran la posibilidad que ofrece el "glosario" de profundizar en los conceptos e identificar y subsanar 
posibles dudas existentes respecto a los mismos; consideran estimulante y enriquecedor la confrontación de opiniones o interpretaciones a la hora de definir términos; aplauden la posibilidad de obtener impreso el glosario, una vez finalizado, para convertirse en material de consulta.

Mientras que todos los encuestados enumeraron aspectos positivos, tan sólo el $40 \%$ se pronunció en los aspectos negativos (el $60 \%$ restante manifestó no encontrar aspectos negativos en el uso de esta herramienta de trabajo). En este caso, la coincidencia en los aspectos negativos identificados ha sido prácticamente total, todos ellos tenían que ver más con la actitud del usuario que con las características propias de la herramienta. De esta forma, apuntan la baja calidad y pertinencia con la materia de algunas entradas, así como la banalidad de algunos comentarios que entorpecían el trabajo en equipo y la construcción del glosario. Igualmente, todos los comentarios coincidían a la hora de acusar el abuso de entradas por parte de algunos que monopolizaban el curso del glosario. En este sentido, los encuestados plantean como mejora establecer unos límites de uso, es decir, un número máximo de entradas por alumno. Por otra parte, se ha comprobado que los estudiantes que han sido más activos en el desarrollo de esta actividad, son también los más motivados a la hora de participar en el aula y los que mejores resultados han obtenido.

\subsection{El juego de rol como herramienta educativa}

La actividad se desarrolló en el marco de la asignatura de cuarto de carrera "Dirección de Cuentas", perteneciente a la Licenciatura de Publicidad y Relaciones Públicas, del curso académico 2011-2012, de la Universidad de Málaga, contando con un total de 68 alumnos matriculados. Según el diccionario de la Lengua Española: "Rol:-Papel que interpreta un actor: desempeñar un gran rol en una representación teatral". Los juegos de rol son una actividad lúdica en la que los jugadores interpretan un papel en una historia cuyo final le es desconocido. A diferencia de otros juegos conocidos en los que el objetivo final (aparte de la diversión) se basa en la victoria, en los juegos de rol se sustituye el concepto de competición por el de colaboración.

Los juegos de rol son útiles para la educación y la formación del alumnado puesto que permiten que el estudiante acceda al conocimiento de forma significativa, permitiendo al docente incentivar la motivación de sus alumnos e introduciendo conceptos, procedimientos y actitudes que de otra manera le resultarían poco atractivas. Asimismo, el incremento de la actividad lectora y de investigación es sustancial; lo que lleva aparejado una mejora en su expresión y riqueza de vocabulario. En lo actitudinal, el juego de rol cumple un papel esencial en lo que a empatía se refiere puesto que el estudiante se pone en la piel de otra persona pudiendo sentir lo que los demás sienten en situaciones que, en principio, le resultan ajenas. También, potencia el apoyo mutuo y la relación con el resto del equipo en términos de igualdad y tolerancia. 


\subsubsection{Descripción de la actividad}

Objetivos planteados. Con esta actividad se pretende que el alumnado realice una dramatización asumiendo un papel de una situación previamente establecida como preparación para enfrentarse a una situación similar en un futuro laboral. Los objetivos principales que se pretenden alcanzar con esta técnica son: Examinar problemas reales en un ambiente laboral determinado; aprender nuevas destrezas ante situaciones concretas y comprender el papel que desempeñan los trabajadores de una agencia; analizar y probar situaciones y tácticas determinadas, sacando fuera temores y ansiedades ante una determinada acción; desarrollar la cohesión grupal y adquirir confianza y competencia individual; aprovechar las posibilidades de las nuevas tecnologías y de las redes sociales para estimular al alumnado en la actividad y profundizar en los conceptos de la asignatura llevándolos más allá del aula.

Recursos empleados y duración de la actividad. Se requiere del estudio del material facilitado por el docente a través de bibliografía, apuntes y pautas. Se facilitará también el uso de las redes sociales, en concreto de Twitter y el hashtag \#dircuen. Asismismo, el docente realiza también el juego de rol, desempeñando el papel de Natalia Casi, Directora de Producto de Mango. La actividad tendrá lugar a lo largo de todo el periodo lectivo, cuatro meses en total.

Diseño de la actividad. La actividad fue presentada a los estudiantes con el nombre: “Concurso Campaña Sweet Dreams by Mango". La tarea consistía en que cada grupo creara una agencia de publicidad y adoptara los roles de sus integrantes para obtener la cuenta y el cliente. El diseño de la actividad es el siguiente:

FASE $1 \rightarrow$ Presentación del Briefing y dinámica de clases: Se les explica a los estudiantes cuál será la dinámica de las clases y se les facilitará el briefing ficticio elaborado con el fin de comenzar la actividad consistente en un concurso de agencias para decidir quién ganará el concurso para trabajar con la cuenta de Mango. Para ello deberán realizar grupos de cinco o seis personas. Se les concede un presupuesto para toda la campaña que abarca desde Septiembre de 2012 a Junio de 2013, de 500.000 euros.

FASE $2 \rightarrow$ Presentación Agencia: Los grupos tendrán que presentarse como agencia habiendo creado su identidad corporativa así como los roles de cada miembro del grupo. Además, deben demostrar por qué se quiere trabajar con ese anunciante y deben exponer los valores de la agencia.

FASE $3 \rightarrow$ Estrategia: Los grupos/agencias deben definir la estrategia a desarrollar en el lanzamiento del producto. Deben exponer los objetivos marcados y las tácticas predefinidas y exponer todas las acciones o gestiones realizados o por realizar.

FASE $4 \rightarrow$ Planificación: Los grupos/agencias temporalizan las acciones para cumplir la estrategia definida. Se explica el presupuesto y agenda de acciones ya negociadas, cerradas y gestionadas. Y, por último, se detallará el cumplimiento del presupuesto, detallando los honorarios para la agencia. 
Previo a las fases 2,3 y 4, los grupos/agencias deberán pedir cita por escrito a Natalia Casi (rol ejercido por la docente) para asignación del orden de presentaciones.

FASE $5 \rightarrow$ Recopilación: Los grupos/agencia entregarán una memoria con todos los pasos seguidos, recogiendo los resultados de todo el trabajo en cada una de las fases.

Función del docente y el discente. A lo largo de toda la actividad, el docente será guía y consejero de todas y cada una de las fases propuestas. Se iniciará la actividad con la aportación del material necesario y controlará el desarrollo de la actividad revisando y tutorizando al alumnado así como resolviendo las dudas que puedan surgirle. El discente deberá ejercer un rol activo en todo el proceso, desempeñando funciones propias de una agencia publicitaria.

Sistema de evaluación de la actividad. La actividad desarrollada se valorará en función de la observación de cada alumno y de su trabajo en el aula así como por las presentaciones y los materiales que se aporten en la presentación de cada fase en la que se divide la actividad. Cada exposición en el aula (adoptando ya el rol tanto el docente como el discente) tendrá una nota y la calificación final será una media ponderada de cada una de las fases.

\subsubsection{Resultados}

Concluida la actividad, se solicitó al alumnado cumplimentar un cuestionario para conocer su opinión respecto a la metodología empleada y la validez en su proceso de aprendizaje. Los resultados de la experiencia han sido muy positivos. El $100 \%$ de los participantes consideran que es una metodología muy acertada y coinciden que han simulado lo que creen se encontrarán al incorporarse al mercado laboral. Resaltan el esfuerzo que les ha costado realizarla aunque consideran que ha merecido la pena. E1 $75 \%$ destacan que a través de esta actividad han podido aprender de sus errores así como a saber comportarse de cara a una exposición en público. Es por ello que el $100 \%$ volvería, sin dudarlo, a repetir.

Respecto al trabajo cooperativo en las TIC, el $35 \%$ de los participantes consideran que se podría mejorar ante todo el uso de la red social Twitter, sacándole más provecho. El $65 \%$ consideran que han contribuido a aportar realismo a la actividad los mails enviados y recibidos de la Directora de Producto (la docente), generando más ilusión por un trabajo bien hecho. La mayoría de los encuestados consideran que la relación teoría-práctica ha sido la idónea e incluso se interesan por adquirir más material sobre la temática abordada.

Como aspectos positivos, el alumnado destaca el realismo concedido a la actividad, la implicación a la que se han visto sometidos, el esfuerzo realizado, el aprendizaje de métodos de búsqueda e investigación, aprender de los demás y de los errores, la idoneidad del trabajo en grupo, aprender a expresarse en público, trabajar la capacidad de improvisación, afán de superación...Una gran mayoría de los participantes considera que es uno de los trabajos de toda la carrera que más les ha aportado y del 
que más han aprendido. Respecto de los aspectos negativos, un $75 \%$ refleja que no ha encontrado ninguno. El otro $25 \%$ hace alusión a nervios, estrés, complejidad del trabajo, falta de tiempo para perfeccionar determinados aspectos...

Respecto a la valoración del docente y la forma de llevar a cabo la metodología y su rol adquirido, elogian su labor; una gran mayoría destacan de ella su dureza y exigencia, su esfuerzo... Consideran que ha sabido motivarlos y enseñarlo a un nivel alto.

\section{Conclusiones}

El aprendizaje cooperativo basado, además, en el uso de los nuevos escenarios virtuales, resulta gratificante, estimulante y enriquecedor para el estudiante. Las TIC se han convertido en un elemento imprescindible de desarrollo de esta sociedad y, por ende, también de las aulas. Estas tecnologías suponen la creación de entornos más flexibles en el aprendizaje y la potenciación de los entornos interactivos favorecen el autoaprendizaje como el colaborativo y el cooperativo; ofrecen nuevas posibilidades de tutorización y orientación a los estudiantes; facilitan una formación permanente e incrementan las modalidades comunicativas. No obstante, a pesar del atractivo que suponen tales entornos interactivos virtuales y de su flexibilidad, no sustituye las virtudes de la clase presencial y las ventajas que supone la interacción personal. De esta forma, entendemos la necesidad de emplear el entorno virtual como complemento y extensión de la transmisión de los conocimientos en el aula.

En cuanto a las herramientas empleadas, ambas han cumplido con los objetivos fijados. La construcción del glosario ha impulsado el pensamiento crítico y la autonomía del estudiante en el proceso de aprendizaje y, como trabajo colaborativo, ha fomentado el trabajo en equipo, el debate y el consenso para la consecución de una meta común. Respecto a la herramienta juego de rol permite acceder al conocimiento de forma significativa, convirtiendo las informaciones en relevantes. La calidad de esta herramienta se basa en la utilidad práctica que el estudiante le encuentra a dicho conocimiento. Con él se desarrollan habilidades de gran importancia como la capacidad de trabajo en grupo, la de toma de decisiones, la de improvisación y resolución de problemas... Permite potenciar habilidades no explotadas en el estudiante y el descubrimiento de intereses y gustos personales así como la motivación y el fomento de la tolerancia y de la empatía. En definitiva, es un juego cooperativo donde no se gana a nadie sino que se juega en colaboración y conjuntamente.

Para finalizar, nos gustaría señalar la necesidad de que, a nivel institucional, se conceda un mayor reconocimiento a la labor del docente a la hora de adaptar las estrategias didácticas a las nuevas exigencias educativas. La virtualización de las asignaturas supone un esfuerzo en tiempo y trabajo no reconocido, ni valorado, desde las instancias superiores. Desarrollar actividades cooperativas en entornos virtuales 
que resulten efectivas y consigan los estándares de calidad necesarios supone una fuerte implicación por parte del profesorado.

\section{Referencias bibliográficas}

BARBERA, Elena (2004). La educación en la red: actividades virtuales de enseñanza y aprendizaje. Barcelona: Paidós.

JOHNSON, D.W. et al. (1999). El aprendizaje cooperativo en el aula. Barcelona: Paidós.

ORGALLO CHANCLÓN, C. (2005). "Técnicas y recursos para profesores que utilizan las Tecnologías de la Información y Comunicación”. En Aplicaciones de la Información y Comunicación. Madrid: MEC, Secretaría General Técnica.

PANIZT, T. and PANIZT, T. (1998). "Encouraging the Use of Collaborative Learning in Higher Education" in FOREST, J.J. (ed), Issues Facing International Education. Garland Publishing. New York.

Artículos en publicación web y documentos en línea:

GIMÉNEZ, Pablo (2009): Los Juegos de Rol: Hacia una propuesta pedagógica. Disponible en: http://dreamers.com/defensadelrol/articulos/propuesta.htm. [9/09/2013]

MOODLE DOCS (2013): Documentación en línea de Moodle. Disponible en: http:// docs.moodle.org/25/en/Main_page [11/09/2013]

ORTEGA, Milagros et al. (2008): El aprendizaje cooperativo. Disponible en: http:// dpto.educacion.navarra.es/publicaciones/pdf/apr_coop.pdf. [6/09/2013]

PÉREZ CASALES, Reinaldo et al. (2008): Algunas experiencias didácticas en el entrono de la plataforma Moodle, en Revista de Informática Educativa y Medios Audiovisuales, Vol. 5(10), 1-10. Disponible en: http://laboratorios.fi.uba.ar/lie/ Revista/Articulos/050510/A1mar2008.pdf. [11/09/2013]

\section{Las autoras}

Rocío Chamizo Sánchez es profesora colaboradora de la Universidad de Málaga, en el departamento de Comunicación Audiovisual y Publicidad y profesora en el Máster Universitario Gestión Estratégica e Innovación en Comunicación. Su investigación se centra en la comunicación desarrollada en la industria del ocio y del turismo, con la elaboración de estudios encaminados a conocer las nuevas formas de comunicación en estos sectores. Es autora del libro "Introducción a la Comunicación Turística. El valor de la imagen en la comercialización de productos turísticos" y es miembro del equipo editorial de la Revista Científica Internacional de Relaciones Públicas, como directora del consejo de redacción. 
María Jesús Fernández Torres es doctora en Ciencias de la Comunicación. En 2004 defendió su tesis doctoral, siendo la doctora más joven de España. Técnico auxiliar en Protocolo, especializada en Comunicación Interna y Externa, funcionaria de carrera y profesora de la Universidad de Málaga. Es autora del libro "Las relaciones públicas como gestión de la comunicación en los movimientos sociales. Análisis de la estrategia comunicativa de las ONG en España". Es secretaria del consejo de redacción de la Revista Internacional de Relaciones Públicas. Ha trabajado en Europa Press y ha sido responsable de comunicación de la Asociación de Consumidores y Usuarios, ASGECO, en Andalucía. 